University of Nebraska - Lincoln

DigitalCommons@University of Nebraska - Lincoln

$9-1-1991$

\title{
Analysis of transport phenomena in the coating of fibers by CVD
}

J H. Scholtz

Laboratory for ceramic and reaction engineering, Department of chemical engineering, State University of New York at Buffalo, New York, 14260.

J E. Gatica

Laboratory for ceramic and Reaction Engineering, Department of chemical Engineering, State University of New York at Buffalo, New York.

Hendrik J. Viljoen

University of Nebraska-Lincoln, hviljoen1@unl.edu

Follow this and additional works at: https://digitalcommons.unl.edu/chemengmatsci

Part of the Materials Science and Engineering Commons

Scholtz, J H.; Gatica, J E.; and Viljoen, Hendrik J., "Analysis of transport phenomena in the coating of fibers by CVD" (1991). Papers in Materials Science. 2.

https://digitalcommons.unl.edu/chemengmatsci/2

This Article is brought to you for free and open access by the Chemical and Biomolecular Engineering Research and Publications at DigitalCommons@University of Nebraska - Lincoln. It has been accepted for inclusion in Papers in Materials Science by an authorized administrator of DigitalCommons@University of Nebraska - Lincoln. 


\title{
ANALYSIS OF TRANSPORT PHENOMENA IN THE COATING OF FIBERS BY CVD
}

\author{
J.H. SCHOLTZ, J.E. GATICA, H.J. VILJOEN and V. HLAVACEK \\ Laboratory for Ceramic and Reaction Engineering, \\ Department of Chemical Engineering, state University of New York \\ at Buffalo, Buffalo, New York 14260, USA
}

\begin{abstract}
Inorganic coated fibers produced by chemical vapor deposition find several techmological applications. In this CVD process, a fiber core is mounted in a reactor between two electrodes. Reactive gases flow over the substrate and react near or on the fiber surface to form the desired coating. Experience has shown that quality deposition call only be obtained over a small range of the governing parameters: these parameters are, however, difficult to control. In the present study, a mathematical model is developed for the reactor configuration under consideration. The different mass and energy transport mechanism are compared and their influence on the reactor performance is evaluated for horizontal reactor arrangements. Numerical results, showing sharp temperature and concentration gradient close to the fiber surface, suggest that heat and mass transfer in this region are controlled by conduction and diffusion mechanisms. In the bulk region, heat and mass transfer via the convection and conduction/diffusion mechanisms can be of equal importance. Numerical results show how bulk properties are affected by both transversal and longitudinal rolls.
\end{abstract}

\section{Introduction}

Over the past decade the advanced composites industry has experienced a dramatic growth, in particular with respect to technological developments. This growth has been driven primarily by the high performance requirements of the aerospace industry, both commercially and militarily. The development of fiber reinforced ceramic and metal matrix composites have been key components. These low density materials exhibit high elastic moduli and tensile strengths which make them attractive in a host of engineering applications [1,2].

Several fibers have been considered for producing composites. Tungsten fibers coated with boron were the first inorganic coated fibers commercially available and have been used for the reinforcement of both polymeric materials and metals, particularly aluninum. These fibers are typically grown at a rate of $14 \mu \mathrm{m} / \mathrm{s}$ in monofilament reactors. An operating temperature of $1260^{\circ} \mathrm{C}$ is typical. Below $1000^{\circ} \mathrm{C}$, very low deposition is obtained while large crytallites are obtained above $1300^{\circ} \mathrm{C}$ [3]. Typical final diameters vary from 100 to $236 \mu \mathrm{m}$. Other inorganic coated fibers commonly considered are $\mathrm{SiC}, \mathrm{Si}_{3} \mathrm{~N}_{4}, \mathrm{BN}, \mathrm{B}_{4} \mathrm{C}, \mathrm{Al}_{2} \mathrm{O}_{3}$ and $\mathrm{TiB}_{2}$.

Table 1. Typical Deposition Reactions

\begin{tabular}{|c|c|c|c|c|}
\hline$\#$ & \multicolumn{3}{|c|}{ Reaction } & Temperature [C. \\
\hline 1 & $2 \mathrm{Ta}+\mathrm{N}_{2}$ & $=$ & $2 \mathrm{TaN}$ & 1000 \\
\hline 2 & $\frac{1}{2} \mathrm{~W}+\mathrm{BCl}_{3}+\frac{3}{2} \mathrm{H}_{2}$ & $=$ & $\frac{1}{x} \mathrm{WB}_{x}+3 \mathrm{HCl}$ & $1800-2000$ \\
\hline 3 & $\mathrm{TiCl}_{4}+2 \mathrm{BCl}_{3}+5 \mathrm{H}_{2}$ & $=$ & $\mathrm{TiB}_{2}+10 \mathrm{HCl}$ & $1000-1300$ \\
\hline 4 & $\mathrm{BCL}_{3}+\frac{3}{2} \mathrm{H}_{2}$ & $=$ & $\mathrm{B}+3 \mathrm{HCl}$ & $1000-1300$ \\
\hline 5 & $\mathrm{CH}_{3} \mathrm{SiCl}_{3}$ & $=$ & $\mathrm{SiC}+3 \mathrm{HCl}$ & $1000-1400$ \\
\hline
\end{tabular}

Examples of deposition reactions that can be performed at atmospheric pressure are shown in 'Table 1 (cf [4]). Examples (1) and (2) demonstrate low a coating can be formed via reaction between the substrate and a constituent in the gas phase. Alternatively, the desired coating can be obtained if two or more gas phase compounds react with each other (examples (3) and (4)). Ceramic coatings can also be obtained from the clecomposition of a single gas phase species (example (5)). 
A versatile reactor configuration suitable for the coating of fibrous substrates is shown schematicaly in Figure 1 $[3,5]$. The fiber core (FC) is monted in a Pyrex tube (PT) between wo electrodes (E). Depending on whether the reactor is operated in a batch or continuous mode, these electrodes can either be copper blocks or pools of mercury. The reactive gases fow over the substrate and react mear or on the fibre surface to form the desirec inorganic coating.

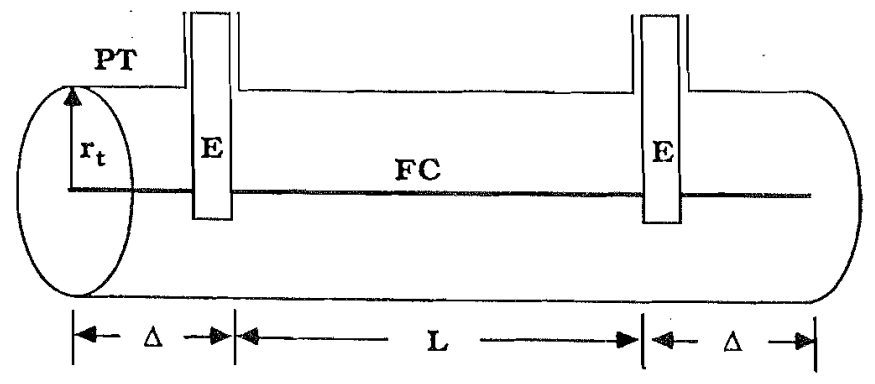

Fig. 1.- Schematic of the CVD reactor.

In the analysis of CVD reactors for the synthesis of fibrous substrates, the published literature is rather limited in its scope. Guinn and Middleman [6] considered the case of a fiber grown in a well mixed reactor. In their study, the effects of reversible reactions and depletion of the reactants on the growth rate of the film were investigated. The presence of thermal or concentration boundary lavers which may exist around the fiber were. however, neglected. Middleman [i] later considered a reactor which is well mixed except for a region close to the fiber surface. Surface and bulk concentrations were related via an overall mass transfer coefficient. which varied axially along the fiber axis. The mass transfer coefficient, however, was derived for the special case of a continuous fiber moving through a stagnant medium. The effects that temperature gradients throughout the reactor, in addition to the acconpanying natural convective flows, may liave on the deposition rate were therefore not taken into consideration. A complete analysis of the gas phase, based on the conservation laws of monentum. heat and mass transfer $(c f,[8,9,10])$ is therefore lacking for the reactor geometry under consideration. Very recently Goköglü and co-workers have taken steps in that direction; in a first work [11] a simplified model assuning a Boussinesq fluid, constant properties, and neglecting end effects and thermo-diffusion was formulated and solved numerically. They found that natural convection was increasingly important for heavier carrier gasses and fingering effects in the temperature field as a. result of feeding the reactants antiparallel to the gravitational field. In the second paper of their series [12];, some of the deficiencies of the previous model were overcome and the improved model was solved numerically, providing also partial comparison with experimental observations. Their results revealed the importance of thermal diffusion effects for the case study of silicon deposition from silane and hydrogen. Both studies followed a similar approach: a two-dimensional model corresponding to vertical reactor arrangements was solved, commercial CFD software was adapted to the geonetry and boundary conditions of the particular problem, essential boundary conditions were used at the fiber surface and end effects were neglected; the boundary conditions at the reactor wall, however, were handled differently in each study. Althongh these analyses are definite steps towards a more comprehensive modeling of this application of CVD technology, some modeling aspects need to be allalyzed more in depth. In previons works, the authors $[13,14]$ addressed vertical reactor arrangements. In those studies thermo-diffusion efferts were neglected and preliminary three-dimensional results were presented.

In the present study, a more in-depth analysis of threedinjensional structures is presented. A more developed mathematical nodel for the reactor configuration under consicleration is formulated. Since the transport phenonena describing the deposition process occur oreseveral space sales the phenomena occurring in the gas phase (external 1.0 the fiber) are decoupled from the phenoment occurring in the solid phase (internal to the fiber). The governing equations are solved numerically over the range of typical operating conditions and results illustrating the relevance of the different design and operating variables are presented. 


\section{Model Formulation}

\subsection{Goveruing Equations}

In describing the gas flow it is important to accont for expansion effects resulting fron large tenperalure gradients in the reaclor chamber. Density dianges clue to pressure variations in the reactor can, however, loe neglected nuder typical CVD conditions. With these assumptions taken into account, the conservation equations for continuity and momentum can be written in a compact form as [15],

\section{Continuity equation}

$$
0=\underline{\nabla} \cdot\left(\frac{u}{\theta}\right)
$$

Momentum balance

$$
0=-\mathrm{Gr}^{\times}\left(\frac{1}{\theta}\right)\left[\frac{\partial \underline{u}}{\partial t}+\underline{u} \cdot(\underline{\Gamma} \underline{u})\right]-\underline{\Gamma}+\underline{\Gamma} \cdot \underline{\underline{\tau}}+\left(\frac{1}{\theta}-1\right) \frac{\underline{g}}{|\underline{g}|}
$$

where the gas has been assuned to follow the ideal gas law. The viscous stress tensor I is given for a newtonian fluid as

$$
\underline{\underline{I}}=\left(\frac{\mu}{\mu^{0}}\right)\left[\underline{\nabla} \underline{u}+(\underline{\nabla} \underline{u})^{T}-\frac{2}{3} \underline{\nabla} \cdot \underline{u} \underline{\underline{I}}\right]
$$

In the energy conservation equation the effects due to changes in pressure, viscous dissipation and Dufour effects may be considered negligible under typical CVD conditions [16]. Furthermore, in the limits of diluted reactants, the themal effects associated with the progress of the chemical reactions can be neglected as well. Thus the temperature distribution is governed by the following equation

\section{Energy balance}

$$
0=-\mathrm{Gr}^{\star} \operatorname{Pe}\left(\frac{C_{p}}{C_{p}^{o}}\right)\left(\frac{1}{\theta}\right)\left[\frac{\partial \theta}{\partial t}+\underline{u} \cdot \underline{\nabla} \theta\right]+\underline{\nabla} \cdot(\mathcal{K} \underline{\nabla} \theta)
$$

'he conservation equation for a diluted species "i", can be written as

\section{Mass balance}

$$
0=-\frac{\operatorname{Pe}}{\operatorname{Le}}\left(\frac{1}{\theta}\right)\left[\frac{\partial x_{i}}{\partial t}+\underline{u} \cdot \underline{\nabla} x_{i}\right]+\underline{\nabla} \cdot\left[\frac{\mathcal{D}_{i m}}{\theta}\left(\underline{\nabla} x_{i}+\alpha \alpha_{i m} x_{i} \underline{\nabla} \ln \theta\right)\right]
$$

where the gas phase reactions lave been neglected. Equation $(5)$ needs to be solved for $i=1, \ldots, S-1$, where $S$ is the number of species present.

Equations (1)-(5) have been vielded non-dimensional by introducing characteristic time and space scales: a cliaracteristic time, $t^{\circ}=R / u^{o}$, and the radius and length of the reactor ( $R$ and $L$ ). Several dimensionless numbers have been introduced: a modified Grashof number $\left(G r^{\star}\right)$, the radial Peclet number (Pe), and the Lewis number (Le). Only a short nomenclature list is presented in this paper, a more complete list can be found elsewhere [13].

\subsection{Boundary Conditions}

A the reactor entrance, essential bonndary conditions can be used to specify the inlet velocity profile. A parabolic flow profile is nsed. The sane condition or, alternatively, the no-stress condition can be applied at the reactor outlet. These conditions are strictily valid only if the reactor extends downstream far beyond the heated substrate. Such an assumption is made here, and temperature and velocity profiles are considered well-developed at the entrance and exit ports. On solid walls, non-slip conditions are applied. In the case of a moving fiber, the fluid velocity on the substrate will be the same as the velocity of the fiber. For the mass balance, diffusion effects at the reactor entrance should be considered. This is done using the well-known Danckwerts' boundary conditions.

On the substrate, heterogeneous reactions have to be taken into account and the resulting boundary condition would be a non-linear set of coupled equations for the mass and entrgy balances. Previons calculations [13], however, revealed that the temperature distribution along the fiber renains approxinately constant between the electrode. This enbbles an additional simplification: the temperature can be prespecified at the fiber surface

The model wouk be completed with the reaction rate expression. This expression will depend on the systen under analysis, for this worli the deposition of $\mathrm{B}$ on $\mathrm{W}$ filanents from $\mathrm{BC}_{3}$ in $\mathrm{H}_{2}$ carrier gas has been selected as case study (cl. example \# 1 in 'lable 1) with the kinetics taken from Carlton a al. [17]. 


\section{Numerical Solution of the Governing Equations}

The governing equations (Eqs. (1)-(5)) are solved numerically by emploving the Cialerkin Finite Element method. lu applying the method. different approaches are followed for t.le two-dimensional (for fully-developer temperature and flow fields) and three-dimensional calculations.

\subsection{Two-dinensional Calculations}

If the axial velocity and temperature gradients, as well as the transient terms, appearing in Equations (1)-(4) are neglected :

- These equations (with the exception of the axial contribution to the monentum balance) collapse to twodimensional expressions depending of the radial $(\eta)$ and the angular $(\phi)$ directions only.

- The continuity, energy and the radial and angular components of the momentum equation decouple from the axial momentum balance and can be solved first.

- Since the velocity profile is assumed fully-developed, from inspection of the momentum equation, it follows that $\frac{\partial P}{\partial \xi}$ has to be constant. By specifying the average axial velocity, this constant can be eliminated from the axial momentum balance. This equation, therefore, collapses to a two-dimensional equations as vell and can be solved numerically once the temperature $(\theta)$ and the radial and angular velocities $\left(u_{\eta}\right.$ and $\left.u_{\phi}\right)$ are known.

In order to solve the continuity, energy and the radial and angular momentum balances, bilinear basis functions are used to approxinate pressure while velocities and temperature are approximated with biquadratic basis functions. The axial momentum balance is solved afterwards employing biquadratic basis to approxinate the axial velocity $\left(u_{\xi}\right)$. Since the physical domain over which the calculations are performed is small (compared to the length of the reactor), a fine enough computational mesh can be used to eliminate the need for upwind techniques.

On a grid consisting of 17 angular and 25 radial mesh points, 6 CPU seconds are used per Newton iteration to solve for $u_{\tau}, u_{\phi}, \mathcal{P}$, and $\theta$. Stating with a default guess for the respective parameters, convergence is obtained typically within $200 \mathrm{CPU}$ seconds on a IBM 3090-600E. The axial component of the momentum equation is linear and converges in 1 iteration. The solution for $u_{\xi}$ can thus be found within 2 CPU seconds.

\subsection{Three-dimensional Calculations}

In the present study it is necessary to perform three-dimensional calculations,

- to solve the species mole fraction distribution for the fully-developed flow situation.

- to obtain a fully three-dimensional solution the continuity, heat and mass transfer equations.

A much smaller bandwidth results as axial coupling is reduced to a span of three nodes only. Since limited compnter storage allocation is a major difficulty in coping with three-dimensional calculations, multi-linear rather that multi-quadratic basis functions are used in theses calculations.

For the fully three-dimensional calculations, a grid consisting of 13 angular, 13 radial and 30 axial mesh points have been emploved. this inplies that 24,456 unknowns have to be solved simultaneously to determine the flow field, and 5,070 unknowns to calculate the species mole fraction distribution.

To determine the mole fraction distribution in the described mesh, $180 \mathrm{CPU}$ seconds are necessary per Newton step if the Jacobian is evaluated and an incomplete inversion of the matrix is performed. Modified Newton steps can be executed in $90 \mathrm{CPU}$ seconds. Since the latter problem is numerically well-conditioned, it is not necessary to perform any false transient steps, and convergence can be obtained within $600 \mathrm{CPU}$ seconds.

Since an iterative solver is used, it should be emphasized that CPU tinies reported above will be dependent on the operating parameters and, for the flow problem, on the time step used in the false transient computations.

For the "incomplete" three-dinensional species mole fraction calculations, a grid consisting of $1 \bar{i}$ angular, 25 radial and 30 axial mesh points have been used. This finer mesh can be afforded since the axial fully-developed flow can be determined in a relatively inexpensive fashion as explained above. To determine the species mole fraction distribution on the latter mesh, 470 CPU seconds are used per Newton step when the Jacobian is evaluated and an incomplete inversion is performed. Modified Newton steps can be executed in $190 \mathrm{CPU}$ seconds. 


\section{Results and Discussion}

Thar solution fields obtainet for the operating conditions shown in Table 2 are presented next

'lable 2. Operating conditions and geometric parameters

\begin{tabular}{||c||cl||}
\hline \hline${ }^{r} \mathrm{BCl}_{3}$ & $5 \times 10^{-2}$ & \\
$r_{f}$ & $1 \times 10^{-4}$ & $\mathrm{~m}$ \\
$R$ & $3 \times 10^{-2}$ & $\mathrm{~m}$ \\
$L$ & $5 \times 10^{-1}$ & $\mathrm{~m}$ \\
$p$ & 100 & $\mathrm{kPa}$ \\
$T^{o}$ & 300 & $\mathrm{~K}$ \\
$T_{f}$ & 1380 & $\mathrm{~K}$ \\
$\left\langle r_{z}\right\rangle$ & $1 \times 10^{-2}$ & $\mathrm{~m} \mathrm{~s}$ \\
\hline
\end{tabular}

In vertical reactors, buoyancy-driven convection manifests as a large convection cell filling the entire reactor length. At low pressures, the contributions of natural and forced convection are equally important, and the effect of natural convection on the temperature field is negligible. The concentration fields are cliaracterized by a thin boundary laver close to the fiber, away from the fiber a linear dependence with the reactor length can be observed. As the pressure is increased, natural convection begins to overshaclow forced convertion, the temperature field becones distorted by the recirculating flows, and "fingering/mixing" effects become visible in the concentration fields. A complete discussion and analysis of vertical configurations can be found elsewhere [13].

\subsection{Incomplete Three-dimensional Formulation}

Streamlines and temperature profiles, as obtained for the fully-developed flow situations, are illustrated in Figure 2. Natural convection manifests itself in the form of two oval cells Iocated at both sides of the fiber (cf. Figure 2 (a)). These cells are strong enongl to result in a plume-like temperature distribution (cf. Figure 4(b)). The contribution of the convection terms in both the nomentum and energy balances is responsible for the lack of vertical symmetry shown by the recirculating cells. Close to the fiber, nevertheless, the isotherms become nearly concentric circles centered around the fiber. This indicates that heat transfer is controlled by conduction in the inner region.

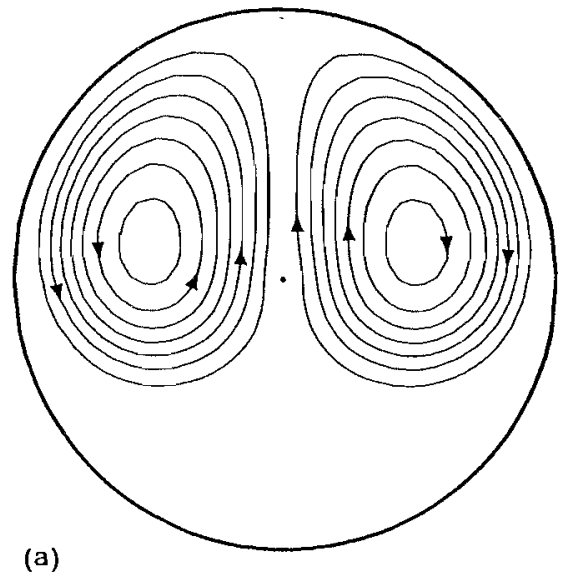

Fig. 2. Streamlines (a) and isotherms (b) as observed in a $(\eta, \phi)$-plane.

Species mole fraction distributions, as ohtained without taking the Soret effect into account, are presented in Figure 3. This figure shows sharp concentration graclients both below and over the fiber. These gradients, however. are sharpened below the fiber and relaxed on top of it due to the effect of natural convection.

Species mole fraction distributions, as obtained taking the Soret effect into account. are presented in Figure 4. This figure reveals once again sharp concentration gradjents both below and on top of the fiber. The boundary laver. however, is more relaxed because the reactants are repelled away from the fiber surface by the thermal diffusion effect. In order to conserve mass. this effect Jeads to high concentration nockets around the fiber. 


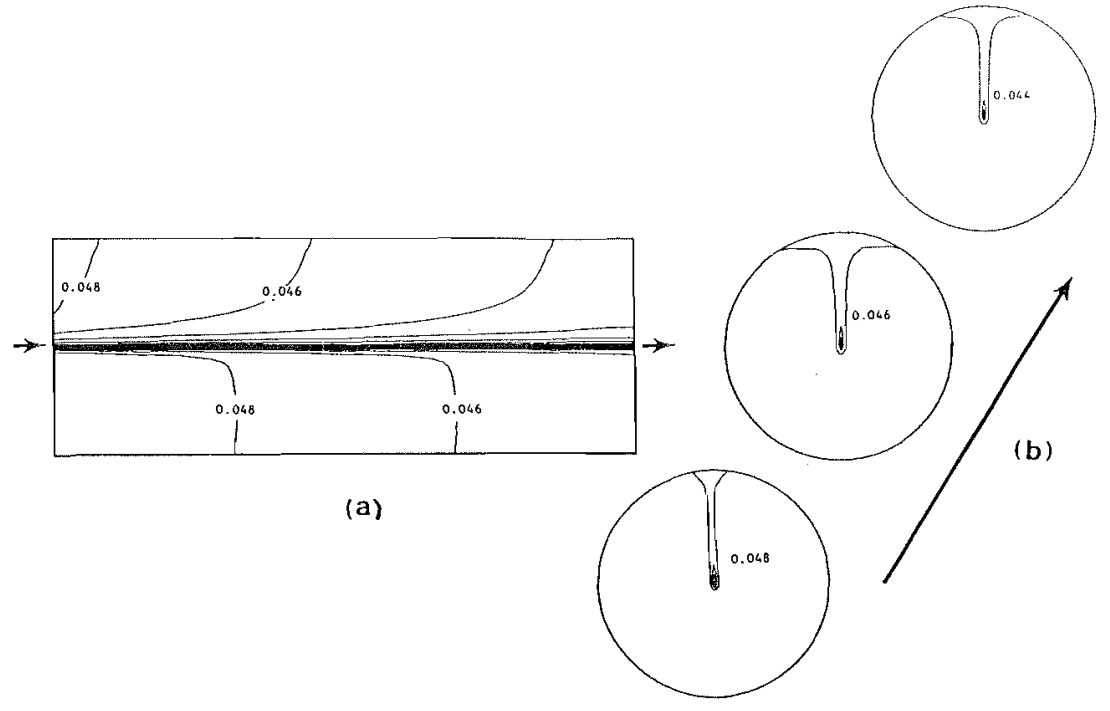

Fig. 3. Species mole fraction distribution of $\mathrm{BCl}_{3}$ for a fully-developed flow problem. Soret effect is ignored. (a) $(\eta, \xi)$-planes a.t $\phi= \pm \pi / 2$ and (b) $(\eta, \phi)$-planes at $\xi=0.1,0.5$ and 0.9 .

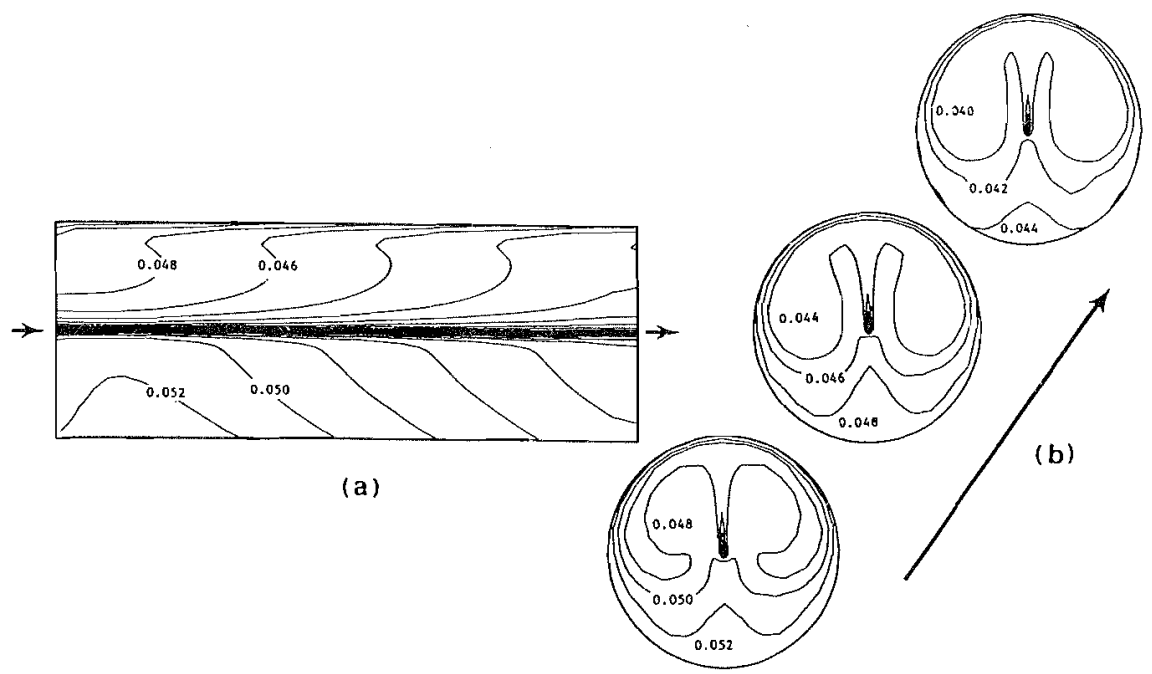

Fig. 4. Species mole fraction clistribution of $\mathrm{BCl}_{3}$ for a fully-developed flow problem. Soret effect is included. (a) $(\eta, \xi)$-planes at $\phi= \pm \pi / 2$ and $(b)(\eta, \phi)$-planes at $\xi=0.1,0.5$ and 0.9 .

\subsection{Complete Three-dimensional Formulation}

Velocity vectors corresponding to a fully three-dimensional calculation are presented in Figure 5. This figure reveals transversal rolls (focated in the vicinity of the electrodes) resulting in backflows at the entrance of the reactor. Over most of the heated section of the sulbstrate, nevertheless, the flow is dominated by longitudinal rolls. As in the case of fully-developed flow, a plume-like temperature in the $(\eta, \phi)$-plane results due to the presence of the strong natural convertion cels. Some qualitative differences between the temperature distributions for the inconplete and fully three-dimensional formulations were obtained. This would suggest that the coarser mesh and linear basis functions used for the latter cose might be unalile to resolve the flow and temperature fields as satisfactorily as the finer mesh and quadratic hasis used in the former case do. 

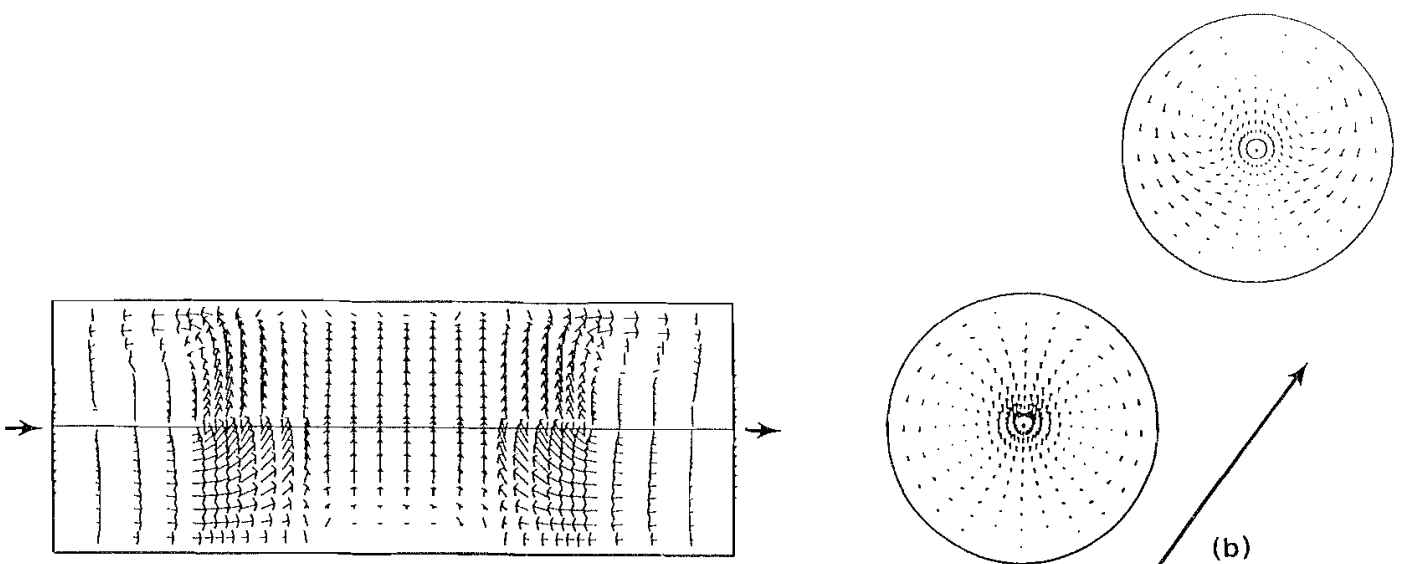

(a)

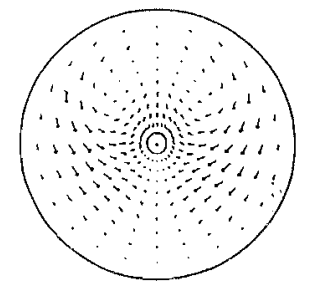

(b)

Fig. 5. Velocity vectors for a fully three-dimensional fow problem.

(a) $(\eta, \xi)$-planes at $\phi= \pm \pi / 2$ and (b) $(\eta, \phi)$-planes at $\xi=0.1,0.5$ and 0.9

As the reaction is taking place on or near the fiber, the temperature and concentration distributions on the fiber itself are of main interest. Anong the different transport mechanisms, interface mass/energy transport is directly affected by the presence of convection. Nusselt numbers for mass and energy can provide an insight in that respect. It is also interesting to analyze the growth rate around and along the fiber. The overall trend in our results has been an increase in the deposition rate with increasing pressure for a constant value of the inlet reactant concentration. This observation, however, should be interpreted with caution since the inlet reactant concentration could be increased at low pressures. The Soret effect appears to reduce appreciably the values of the Nusselt numbers from the values obtained with the Soret effect neglected. Its effect on the deposition rate, however, is not as significant as expected. This is due to the particular case study chosen; both the reactants $\left(\mathrm{BCl}_{3}\right)$ and by-products ( $\mathrm{HCl}$ ) are repelled away from the susceptor. The effect for a different reaction system (or carrier gas, cf. [12]) can be quite different. The deposition rate, however, remains lower on top of the fiber than the one observed for the lower part of the fiber. This can be explained as an effect of the angular decrease (increase) of $\mathrm{BCl}_{3}$ ( $\mathrm{HCl}$ ). In addition, an axial decrease in the deposition rate is observed; most likely attributable to the consumption of the reactants. Finally, an interesting fact should be mentioned: preliminary calculations including pressure dependence of the gas diffusivities revealed that decreasing the reactor pressure might lead to increasing non-uniformity in the deposition race. This supports the observation that some of the results obtained for different CVD configurations (e.g. the waler reactor) cannot be extended to the configuration under study.

\section{Acknowledgements}

The authors want to acknowledge financial support provided by grants from the National Science Foundalion (Grant \# CBT 88-13918) and the U.S. Navy/DARPA (Cirant \# N00014-89-J-3203). The computations supporling 1.his rescarch were performed on an IBM $3000-600 \mathrm{E}$ supercomputel using the Comell National Supercomputer Facility, a resonrce of the Center for Theory and Simulation in Enginering at Cormell University, which receives major funding from the National Science Foundation and the IBM Corporation, with additional support from the State of New York and members of l he Corporate Research Institute. 


\section{References}

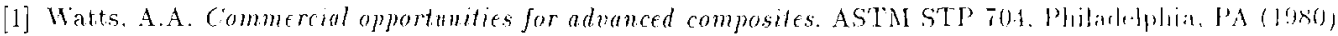

[2] D)icarlo, J.A. J. Wet. 37 (1985) $44-19$.

[3] Morley, J (i. High-Performance Fibre Composites, Academic Press, LOndon (198T).

[4] Blocher. J.M.: Browning, M.F. and Barret, D.M. Mater. Sci. Res 17 (1984) 299-315

[5] Krukonis, V. in Boron and Refractory Borides (Ed. V.I. Matkovich), pp. 5] i-540, Springer-Verlag. Berlin $(197 \mathrm{i})$

[6] Guinn, K. and Middleman, S. J. Cryst. Growth 96 (1989) 589-595.

[7] Middleman, S. J. Cryst. Growth 98 (1989) 810-816.

[8] Coltrin, M.E; hee, R.J. and Miller, J.A. J. Electrochem. Soc. 133 (1986) 1206-12I3.

[9] Moffat. H.K and Jensen, h.F. J. Electrochcm. Soc. 135 (1988) 459-471.

[10] Kileijn, C.R. and Hoogendoorn, C.J. Chem. Engng. Sci 46(1) (1991) 321-334.

[11] Coköglü, S.A.; Amold, W.A.; Tsui, P. and Chait, A. in Transport Phenomena in Manufacluring (Eds. P.A. Pfund, J.A. Khodadi and S.l. Guger), pp. 9-20, ASME FED, Vol. 90 (1989).

[12] Coköglü, S.A.; Kuczmarski, M.; Veitch, L.; Tsui, P. and Chait, A. 11th Int. Conf. on Chemical Vapor Deposition (CVD-XI), Seattle, WA (1990).

[13] Scholtz, J.H. and Hlavacek V. J. Electrochem. Soc. 137 (1990) 3459-3469.

[14] Scholtz, J.H.; Gatica, J.E.. Revankar, V.; Viljoen, H.J. and Hlavacek V. Chem. Engng. Sci. 45(8) (1990) $2543-2550$.

[15] Bird. R.B.; Stewart, W'E. and Lightfoot, E.N. Transport Phenomena, Wiley, New York. NY (1960).

[16] Jenkinson, J.P. and Pollard, R. J. Electrochem. Soc 131 (1984) 2911-2917.

[17] Carlton. H.E.; Oxley, J.H.; Hall, E.H. and Blocher, J.M. in Proc. of the 2nd Int. Conf. on CVD (Eds. J.M. Blocher. Jr. and J.C. Withers), pp. 209-225, The Electrochem. Soc. Pennington, NJ (1970).

\section{Nomenclature}

$C_{p}$
$D_{i m}$
$k$
$r_{f}$
$T$
$\underline{u}$
$x_{i}$
$\langle\cdot\rangle^{\circ}$
$(\cdot)^{\circ}$

\section{Greek Symbols}

$\alpha_{i m}$
$\xi$
$\eta$
$\phi$
$\mu$
$\rho$
$\theta$
$\mathcal{P}$
$\mathcal{D}_{i m}$
$\mathcal{K}$

Dimensionless Numbers

$\begin{array}{ll}\text { Gr* } & \text { Modified Cirshof number. } \\ \text { Le } & \text { Lewis number, } \\ \mathrm{Pe} & \text { Peclet number, } \\ \mathrm{Pr} & \text { Prandil number. }\end{array}$

Specific heat,

Diffusivity of component "i" in the mixture,

Thermal conductivity,

Outer radius of the fiber,

Temperature,

Dimensionless velocity vector,

Mole fraction of component "i".

Average value.

Reference value.

Thermal diffusion coefficient for component "i"

Dimensionless axial coordinate,

Dinnensionless radial coordinate,

Angular coordinate.

Dynamic viscosity,

Density,

Dimeusionless temperature,

Dimensionless pressure,

Dimensionless diffusivity,

Dimensionless thermal conductivity,
$\mathrm{J} \mathrm{kg}^{-1} \mathrm{~h}^{-1}$.

$\mathrm{nt}^{2} \mathrm{~s}^{-1}$.

$\mathrm{J} \mathrm{m}^{-1} \mathrm{~s}^{-1} \mathrm{~h}^{-1}$.

$\mathrm{m}$.

K.

$\underline{u}=\underline{u}^{\prime} / u^{\circ}=\left(u_{\eta}, u_{\phi}, u_{\xi}\right)$.

$$
\begin{aligned}
& \xi=z / L . \\
& \eta=r / R . \\
& \mathrm{kg} \mathrm{m}^{-1} \mathrm{~s}^{-1} . \\
& \mathrm{kg} \mathrm{m} \mathrm{m}^{-3} . \\
& \theta=T / T^{o} . \\
& \mathcal{P}=p R / \mu^{o} u^{\circ}+\eta \sin \phi . \\
& \mathcal{D}_{i m}=D_{i m} / D_{i m}^{o} . \\
& \mathcal{X}=k / k^{\circ} .
\end{aligned}
$$

